\title{
Experiencias en territorio: vulnerabilidad y problemáticas sanitarias en barrios periurbanos de Paraná (Entre Ríos - Argentina)'
}

\section{Experiences in territory: vulnerability and health problems in peri-urban neighborhoods of Paraná (Entre Ríos - Argentina)}

Leila Martina Passerino

(D) https://orcid.org/0000-0002-2343-3445

E-mail: leilapasseळgmail.com

Centro de Investigación y Transferencia Rafaela (UNRaf-CONICET), Santa Fe, Argentina.

\section{Correspondência}

Bv. Pres. Julio A. Roca 989, Rafaela, Santa Fe, Argentina - CP: 2300

1 Agradezco a las instituciones que financiaron esta producción: CONICET, INC, UBA y UNER de Argentina.

\section{Resumen}

En el siguiente artículo indagamos problemáticas de salud/enfermedad a partir de la experiencia de sujetos que habitan barrios periurbanos de la ciudad de Paraná (Entre Ríos, Argentina). A partir de una etnografía colaborativa durante los años 2017-2018 -que incluyó entrevistas en profundidad, registros audiovisuales e investigación-acción a vecinos de barrios periurbanos de la ciudad de Paraná, visita a Centros de Salud, entrevistas a profesionales y personal administrativo de los mismos, como de algunas Organizaciones que trabajan en promoción de la salud y prevención de dolencias y/o agravios - se indagan los procesos de determinación social de la salud-enfermedadmuerte en territorios vulnerables. A partir de cierta reconstrucción, profundizamos fundamentalmente en las consecuencias y dinámicas que encarna en la vida de quienes habitan el territorio la existencia de un basural a cielo abierto, el cual resulta epicentro para abordar problemáticas sanitarias. Ahondar en las determinaciones, supone un recorrido por situaciones socio-históricas, políticas y culturales de este territorio que hacen mella en las corporalidades, en los modos de comprender los procesos de salud/enfermedad y vivenciarlos. Palabras clave: Determinación; Vulnerabilidad; Territorio; Procesos Salud/Enfermedad; Basural. 
In the following article we investigate health / illness problems from the experience of subjects who live in peri-urban neighborhoods in the city of Paraná (Entre Ríos, Argentina). Based on a collaborative ethnography during the years 2017-2018, which included in-depth interviews, audiovisual records, and action research with residents of peri-urban neighborhoods in the city of Paraná, visits to Health Centers, interviews with professionals and their administrative personnel. As with some Organizations that work in health promotion and disease prevention, the processes of social determination of health-illness-death in vulnerable territories are investigated. After a certain reconstruction, we fundamentally delve into the consequences and dynamics that the existence of an open-air dump embodies in the lives of those who inhabit the territory, which is the epicenter for addressing various health situations. Deepening in the determinations, involves a journey through socio-historical, political and cultural situations of this territory that make an impression on the corporalities, in the ways of understanding the processes of health / illness and experiencing them.

Key-words: Determination; Vulnerability; Territory; Health/Illness Processes; Dump.
En el siguiente artículo indagamos problemáticas de salud a partir de la experiencia de sujetos que habitan barrios periurbanos de la ciudad de Paraná (Entre Ríos, Argentina). Anticipamos que estos aspectos se inscriben en una perspectiva de derechos que requiere pensar la salud en territorio. Esta clave, implica a la integralidad en salud, como campo en disputa, atravesada por componentes históricos, sociales, culturales, económicos y políticos que exceden tanto normativas jurídicas como un abordaje de los procesos de enfermedad como instancias objetivas, ahistóricas, capaces de escindirse de los cuerpos que las portan, sufren y conviven.

La construcción de problemas sociales desde la perspectiva de la territorialidad supone cartografiar la singularidad de los espacios, que circunscribimos en este caso a barrios periurbanos de la ciudad capital de la Provincia de Entre Ríos², Paraná, ubicada sobre el margen izquierdo del río que le da su nombre en Argentina. San Agustín, Balvi, Las Flores, La Floresta, Antártida, San Martín son algunos de los barrios que forman parte del espacio abordado en este estudio, ubicados en la periferia, al sudoeste de esta ciudad. Barrios caracterizados por algunos de sus pobladores como "marginales" y "discriminados", que comparten problemáticas de diversa índole. Se caracterizan por un alto nivel de pobreza, precariedad en las viviendas -muchas de ellas con paredes de chapa y techos de loca- sin servicios básicos como cloacas y agua potable; con calles de tierra. Además, son barrios aledaños a un basural a cielo abierto (Volcadero), que imprime dinámicas propias y configura vulnerabilidades en materia de salud. Es el vertedero de todos los residuos que se generan en la ciudad capital y tiene una existencia aproximada de 100 años. En sus orígenes, resultaba un lugar alejado de la ciudad que pronto se fue poblando y adquiriendo otras dimensiones. A la luz de las políticas neoliberales y excluyentes de la década del noventa, y en un

\footnotetext{
2 La provincia de Entre Ríos está ubicada en el área centro-este de la República Argentina, al sur del Continente Americano. Cobra importancia por su posición geográfica estratégica que comprende un paso obligado en el eje norte-sur de la República Argentina con Brasil y el eje este-oeste comprendido por el Corredor Bioceánico que une Uruguay y Chile (Gobierno de Entre Ríos, 2019). Paraná, su capital, tiene una población aproximada de 340.000 habitantes sobre una superficie de 1.974 km² (INDEC, 2010).
} 
contexto de empobrecimiento, exclusión y pérdida de empleo en Argentina, el volcadero, como se lo reconoce, resulta epicentro para muchas familias que, excluidas del empleo formal, se valen del mismo para generar algún ingreso u obtener elementos para su consumo.

A partir de una etnografía colaborativa (Lawless, 1993; Lassiter et al., 2004; Lassiter, 2005; Rappaport; Rodriguez, 2007) durante el período 2016-2019-que incluyó entrevistas en profundidad, registros audiovisuales e investigación acción a vecinos de barrios periurbanos de la ciudad de Paraná, visita a Centros de Salud, entrevistas a profesionales y personal administrativo de los mismos ${ }^{3}$, como de algunas Organizaciones que trabajan en promoción de la salud y prevención de dolencias y/o agravios, como la Casa de Atención y Acompañamiento Comunitario (CAAC)- se indagan los procesos de determinación social de la salud-enfermedad-muerte.

Las reflexiones producidas forman parte de un extenso trabajo desde el cual hemos podido reconstruir algunos argumentos en coproducción con quienes habitan estos espacios, junto con el equipo de investigación e intervención ${ }^{4}$. En términos metodológicos, en un primer ciclo de trabajo etnográfico, se identificaron informantes clave, registros de campo, se realizaron entrevistas no dirigidas y observaciones participantes, lo cual permitió jerarquizar las prioridades locales, identificar categorías emic y establecer alianzas con organizaciones y redes en las comunidades (Luxardo, Sassetti, Bello, s/f). Junto a las comunidades se establecieron luego diversos objetivos, revisando intereses comunes entre los objetivos académicos -de investigadores externos-y los del lugar -investigadores internos y que trabajan en instituciones como organizaciones de base, centros de salud y miembros de la comunidad-, aspecto facilitado también por los intercambios y presencia en la organización de base CAAC. El trabajo colaborativo con las comunidades se estableció con dos grupos sociales fundamentalmente: trabajadores informales en el basural a cielo abierto y personas abocadas a la prevención de problemáticas de consumo en esos barrios.

La etnografía colaborativa enfatiza la construcción de conocimiento, reconociendo los procesos de producción, involucrando y haciendo partícipes a quienes encarnan esas vivencias como parte inherente del mismo. Se trata así de acentuar la colaboración en el mismo proceso etnográfico en vez de ocultar estas relaciones (Rappaport; Rodriguez, 2007), produciendo un ida y vuelta que se reintegra en el proceso de trabajo de campo (Lassiter, 2005) y que excede su institución como "dato". Recuperamos relatos a través de entrevistas ${ }^{5}$, registros etnográficos y desde las propias microhistorias. Reflexionamos codo a codo con quienes habitan estos espacios desde un enfoque que hunde sus raíces en las discusiones que encaran exponentes de la Medicina Social y Salud Colectiva. Procuramos, de este modo, articular los procesos socioculturales con las dimensiones de salud/enfermedad/atención/cuidado mediante una construcción de conocimiento que involucra y hace partícipes a quienes habitan la cotidianidad del territorio indagado.

Estructuramos el artículo en tres apartados. En el primero, esbozamos los lineamientos

\footnotetext{
3 Se trabajó con 3 centros de Salud ubicados dentro del área de estudio, que no se mencionan para resguardar la confidencialidad, y que contaban con trabajadores e investigadores internos, los cuales garantizaron el trabajo colaborativo frente a los intereses y prioridades locales.

4 Los resultados publicados en este artículo forman parte del libro $X X X$ actualmente en elaboración, que concentra los resultados de una serie de investigaciones sobre cáncer articuladas entre sí financiadas por XXX durante los años 2016-2019. Se trata de un proyecto mayor que involucra una amplia gama de métodos cualitativos, cuantitativos, participativos. Por razones de espacio, no indagamos en profundidad aquí sobre las estrategias y métodos desarrollados, aunque esto se encuentra en la publicación mencionada en distintos capítulos de esa obra. Asimismo, vale mencionar que muchos de los sujetos/partícipes de la investigación son coautores y/o autores de capítulos y han participado en el análisis de los datos como en las diferentes etapas de la investigación llevada a cabo bajo la dirección de la Dra. Natalia Luxardo.

5 Las entrevistas, contaron con un Consentimiento Informado, en el cual se avaló la participación, primero comentado de modo oral y luego entregado para su firma. Incluyó cláusulas de confidencialidad y derechos personales de privacidad, asegurándonos que los nombres y datos personales de las participantes no fueran revelados. Se contó además con los resguardos éticos considerados en los "Lineamientos para el comportamiento ético en las Ciencias Sociales y Humanidades” del Comité de Ética del Consejo Nacional de Investigaciones Científicas y Técnicas (CONICET, 2006).
} 
teórico-epistemológicos respecto a cómo leer la noción de territorio. En el segundo, cartografiamos algunas características propias del espacio estudiado, profundizando en la existencia de un basural a cielo abierto el cual caracteriza e imprime dinámicas propias en el territorio estudiado. En el último, recuperamos la noción de territorio urbano de sacrificio y de determinación, para dar cuenta de las complejidades y circuitos de vulnerabilidad que atraviesan la vida en el territorio estudiado, profundizando dinámicas y también paradojas.

\section{Algunas consideraciones teóricas sobre territorio}

La noción de territorio, pese a las distintas derivas teóricas, asume una primera diferencia con la concepción ligada al espacio físico-geográfico, delimitable y delimitado, fijado con antelación, estable y autodefinido. Históricamente, esta comprensión se asoció a las guerras por el control de espacios físicos, a los fines de identificar y disponer tanto de sus recursos naturales como humanos (Borde; Torres-Tovar, 2017). En esta línea, la geografía crítica diferencia la noción de lugar de la de territorio, entendiendo al lugar como la ubicación geográfica de las personas en un tiempo determinado (Spinelli, 2016). Aun así, dentro de la disciplina, las acepciones también cambian y, dentro de la denominada geografía de la vida, el lugar asume un lugar próximo al que proponemos desarrollar a partir de la noción de territorio, dado que supone un complejo proceso de apropiación por identificación, involucrando aspectos afectivos y simbólicos, a través de la experiencia práctica de quienes habitan esos espacios (López; Michelli, 2016). Como argumentan estos autores desde los cuales adscribimos, el territorio implica una experiencia espacial, como un "saber habitar", que recupera la cotidianeidad. Un "lugar donde se 'encuentran' en una dinámica compleja la vivencia subjetiva y la producción y reproducción de las estructuras sociales” (López, Michellin, 2016 , p. 5o).
En tanto devenir histórico, el territorio involucra condiciones y modos devida ${ }^{6}$ de los conjuntos sociales, siempre vinculado a relaciones de poder, un "espacio relacional-simbólico, en el cual actúan capitales y campos que se crean, recrean y pueden extenderlo" (Spinelli, 2016, p. 16o). A su vez, actúa como categoría densa para expresar, en palabras de Porto-Gonçalvez, modos de apropiación -territorialización-, los cuales habilitan a crear condiciones para la construcción de identidades -territorialidades- anudadas a procesos históricos: "Estamos lejos pues de un espaciosustancia, y sí ante una triada relacional territorioterritorialidad-territorialización. La sociedad se territorializa siendo el territorio su condición de existencia material” (Molina Jaramillo, 2018, p. 6). El territorio condensa las tramas estructurales desde donde se producen modos posibles de territorialización, como tramas laberínticas propias de lo social (Spinelli, 2016).

El territorio recupera procesos de identificación, de imaginación del mundo, como modos de concebir y comprender las prácticas de los sujetos en tramas sociales complejas, donde se articulan no solo racionalidades, sino indefectiblemente, aspectos afectivos con el espacio vivido, desde donde se siente y comprende, desde donde también se delimitan oportunidades y transformaciones posibles. De modo que el dinamismo resulta una característica intrínseca, y su analítica solo puede pensarse como una construcción política que involucra decisiones ancladas en las interacciones entre quienes habitan esos espacios y entre quienes procuramos caracterizar ciertos procesos, una decisión ${ }^{7}$. Como refiere Spinelli (2016), el territorio es una construcción humana, inacabada, en permanente movimiento y transformación, que puede ser hogar o cárcel también para los sujetos ${ }^{8}$. Estas dinámicas territoriales, por fuera de una visión romantizada, lo alojan como hogar, transitado, ocupado, significado, disputado, desde el cual se construyen distintos procesos de subjetivación, lazos comunitarios, prácticas de cuidado y también resistencias frente a distintos

6 Vale la pena recuperar el trabajo de Polo Almeida (2016) quien reconstruye históricamente la noción y sistematiza su desarrollo en el campo de la Salud Colectiva Latinoamericana, diferenciando, asimismo, la propuesta anglosajona de la Ecología Política.

7 En esta línea, es que puede establecerse una cercanía y familiaridad con la noción de campo antropológico (Guber , 2005).

8 Para profundizar estos aspectos desde un análisis de las políticas públicas en el Gran Buenos Aires, ver Chiara (2012, 2016). 
tipos de vulneración, tanto a partir de experiencias singulares como colectivas. Simultáneamente, opera muchas veces como cárcel -territorios "marginalizados", “discriminados”, como aluden algunos de sus pobladores, o zonas de sacrificio, como posteriormente aludiremos- en el cual se reproducen desigualdades y diversos circuitos de vulnerabilidad, como referiremos en los próximos apartados.

La dimensión territorial ocupa un lugar central no solo para pensar políticas sanitarias que atiendan a las desigualdades y fenómenos estructurales, sino también en términos inversos, como refiriera Chiara (2016), construir territorialidad frente a las demandas, necesidades y proyectos que la propia comunidad delinea. De aquí que los modos de vida ${ }^{9}$ y sentidos de apropiación de los territorios constituyan elementos centrales para el campo de la Medicina Social y Salud Colectiva (Breilh, 2003; Breilh, 2013; Almeida Filho, 200o; Laurell, 1994). Los modos de vida integran lo colectivo en lo cotidiano como modos de hacer, pero también de significar, para sostener o también trasformar las prácticas. El modo de vida anula entonces la dicotomía individual-colectivo, dado que los sujetos asumen sus movimientos en los marcos de la propia comunidad, con sus posibilidades y tradiciones, una clave necesaria para comprender los procesos de salud/enfermedad/atención/cuidado como los modos específicos de vivir, padecer, enfermar, morir, cuidar. Territorio, vivencia y significación se anudan de modo inherente, caracterizados por el dinamismo que produce el movimiento de territorialización.

\section{Rasgos del territorio: problemáticas, carencias y "volcadero"}

Si pensar en territorio implica una experiencia espacial, un saber habitar la cotidianidad, la etnografía colaborativa resultó crucial para construir colectivamente las problemáticas más acuciantes para sus pobladores e investigadores internos. Las carencias más evidentes forman parte de la infraestructura de estos barrios, por ejemplo respecto a la falta de cloacas o a la presencia de "cloacas reventadas" que desbordan; desembocan con toda la "mugre" porque están mal realizadas las caídas; se tapan porque cae basura. Su permanencia al descubierto provoca accidentes en niños, que juegan en la vía pública, exponiéndolos a peligros que pueden ser prevenidos. Como expresa un poblador del Barrio Balvi, esta situación directamente se cataloga como "inhumana":

Me afecta a míy donde vivo y a mucha gente, porque bueno, para mí es como yo le decía a él hoy, para mí no está bien que hayan reventado una cloaca donde están jugando las criaturas y las personas mayores. Para mí esto es inhumano y bueno...ya va a hacer un año y tuvimos un accidente con una nenita, con mi sobrina. Estaba la tapa, la tapa estaba afuera, la tapa estaba...le habían sacado la tapa y...eso corre peligro de una criatura que... (Poblador Barrio Balvi, 2018)

Asimismo, los pobladores reconocen las condiciones de precariedad de las viviendas, construidas muchas de ellas a partir de chapas y otros elementos que encuentran en basurales a cielo abierto, como tablas y plásticos. El acceso a servicios básicos también resulta problemático. Como relatan algunos trabajadores de los centros comunitarios del barrio, entre un 80 y $90 \%$, se encuentran "colgados" al tendido del sistema de iluminación pública. El suministro de agua, sobre todo en verano, es escaso y muchas veces se "corta", de modo que los vecinos y vecinas deben anticiparse para juntar agua en tachos. El acceso a gas, tanto para cocinar como para calefaccionar, también es un recurso escaso. En la mayoría de las casas, la garrafa de la cocina representa la única instalación de gas y muchas veces en invierno lo que hacen es...si se quedan con gas, prenden (...) adentro de la casa un tacho o algún contenedor que le permita calentar un poco la casa (Trabajadora del CAAC, 2018). Esta situación conlleva peligros asociados a tener fuego cercano a productos inflamables presentes en los hogares y la posibilidad de incendios.

En términos socioeducativos, el analfabetismo resulta una problemática básica registrada por algunos

9 La distinción con el estilo de vida, asociada a prácticas y decisiones individuales voluntaristas, es crucial para introducir la noción de modos de vida en tanto praxis personal y colectiva (Menéndez, 2009) con diversas implicancias para la Salud Pública (Almeida Filho, 20oo). 
de los profesionales que trabajan en los Centros de Atención Primaria y en las Organizaciones Barriales. La deserción escolar forma parte de la necesidad de muchos jóvenes de utilizar ese tiempo para trabajar, pero también, en oportunidades, para generar ingresos asociados al consumo de sustancias, fundamentalmente drogas y alcohol, que se inicia alrededor de los 11, 12 años de edad. Los embarazos son otra de las razones por las cuales muchas adolescentes abandonan las instituciones educativas para los cuidados y crianza de los bebés, tarea delegada exclusivamente a las mujeres.

En términos salariales, muchas de las personas que habitan los barrios tienen el Salario Social Complementario (SSC) que representa la mitad del mínimo y móvil, para sostenerse todo el mes, o se valen de trabajos precarizados. Una de las principales fuentes de ingreso, que comparten quienes habitan el territorio, forma parte del trabajo en el volca o volcadero -desde la categoría nativa-, el basural a cielo abierto que comparten estos barrios periurbanos de la ciudad de Paraná. Como mencionáramos en la introducción, pese a que se ubicó inicialmente en las periferias a la ciudad capital, la expansión demográfica -acompañada de políticas neoliberales, el empobrecimiento, la exclusión y la pérdida de empleo-promovió el aumento de población en la zona aledaña, constituyéndose en epicentro para muchas familias que, excluidas del empleo formal, se valieron y valen del mismo para generar algún ingreso u obtener elementos para su consumo.

$\mathrm{Al}$ volcadero se lo concibe como espacio de trabajo para los "clasificadores" o para el "cirujeo", donde diferentes generaciones van aprendiendo del "vivir de la basura", lo cual supone pasar largas jornadas, de entre 12 y 14 horas para tener algún rédito. La "clasificación" exige, por un lado, separar la basura como papel, plásticos o metales para luego comercializar estos elementos y comprar comida. También se reconoce una cooperativa de gente que recicla y selecciona a partir de quemar basura. Esta práctica se ha extendido, dado que permite recuperar metales entre los desechos de una manera más rápida. Por otro lado, están quienes son identificados como "cirujas", es decir, gente que busca en la basura productos para su consumo directo y diario, como actividad de extrema necesidad ante falta de otras alternativas. Como expresa una de las mujeres que participa en el CAAC del barrio:

No es lo mejor y no es bueno, pero...si no hay para comer, de alguna manera te la tenés que rebuscar y hay mucha gente acá que antes de salir a robar, prefiere ir a cirujear que salir a robar. (Pobladora Barrio San Martin)

El volcadero resulta un espacio compartido por personas de muchas edades, adultos/as mayores, jóvenes, niños/as y adolescentes. Muchos de los niños/ as en edad escolar, se dirigen al Centro Comunitario a tomar la "copa de leche", como una especie de "corte" o "recreo" del trabajo cuando son más grandes. También es vivido como juego o ayuda a sus padres, cuando son más pequeños/as. Asimismo, algunos adultos o jóvenes padres llevan a sus hijos/as dado que las tareas de cuidado se dificultan:

Pongalé, yo tengo mi nene que tiene 4 años. Pongale, yo voy al volca a trabajar y lo llevo. (Pobladora Barrio San Agustín)

Y...hay mucha gente, para la gente eso es un trabajo. Por eso te digo, las criaturas acá, hay gente que no tiene quien cuide de sus hijos y lo lleva y ya esa criatura nac, ya viene con...creciendo con eso de que los padres van al volcadero, se rebuscan la vida ahí y yo voy a la par de ellos. (Vecina del Barrio San Martín, participa del CAAC)

El volcadero representa un lugar de trabajo para numerosas personas y familias. Pero también se esgrime como espacio de disputa entre quienes lo habitan a diario. Existen códigos y modos de organización que permiten advertir al territorio como espacio de poder. Los camiones se asignan, es decir, en las descargas: "cada basura tiene su dueño".

Puede decirse que el volca o volcadero imprime dinámicas propias en el territorio estudiado: en las prácticas de quienes habitan, en la organización, distribución y modos de generar recursos, en las decisiones y oportunidades socioeducativas y en los modos en que la población atraviesa, vivencia y significa los procesos de salud/enfermedad/ atención/cuidado. 


\section{Territorios urbanos de sacrificio: complejidades, violencias y disyuntivas para la acción sanitaria}

El abordaje anclado al territorio, dentro del campo de la salud y de la salud pública, recupera los procesos de producción y reproducción social como base de la determinación social de la saludenfermedad-muerte (Borde; Torres-Tovar, 2017). Comprender los modos y escenarios donde transcurre la vida de quienes habitan los barrios analizados representa una base insustituible para comprender las desigualdades e inequidades, las diversas formas de enfermar, los modos de sufrimiento y las posibilidades de bienestar (Breilh, 2010). Pensar en salud implica reorientar las acciones a los procesos de determinación social que son los que reproducen ciertas condiciones histórico-territoriales: "Es decir, el territorio produce social y biológicamente la vida, indefectiblemente ligada con la salud" (Borde; TorresTovar, 2017, p. 271).

La corriente de la Salud Colectiva problematiza las causalidades unidireccionales para abordar la determinación social de la salud como campo complejo y multidimensional que obliga a considerar al territorio, en sus relaciones productivas y sociales, como parte de un sistema de acumulación de poder y cultura, como parte de un orden estructural e histórico (Casallas-Murillo, 2017). La determinación social de las desigualdades, en los procesos de salud/enfermedad, refiere a la multiplicidad de aspectos que inciden en los modos en que las personas viven, enferman, se cuidan, mueren y también acceden a la atención: jerarquías sociales que definen el acceso y la calidad de los servicios de salud, las condiciones de vida y los hábitats, el acceso a la educación, los consumos problemáticos, las posibilidades y condiciones de empleo, las diferentes violencias participantes, entre otras determinaciones que inciden y resultan “expresión íntima de procesos histórico-socialesespaciales en 'sujetos socio-históricos que enferman' (Hernández; Quevedo, 1992)" (Borde; Torres-Tovar, 2017, p. 272). La determinación discute así con propuestas que producen relaciones de causalidad entre factores o visiones dicotómicas -como individuo/sociedad- para asumir la historia, las contradicciones, los procesos sociales: "Más que 'ex-posición' a riesgos externos, los sujetos sufren procesos de ‘im-posición' de condiciones de vida (Hernández, 2008, p. 9). Las problemáticas de salud no pueden ser pensadas en términos de individualidades, sino que forman parte de las mismas dinámicas territoriales. Como bien afirma Spinelli, "El territorio nos demuestra que la epidermis no es el límite del individuo y que si vamos más allá de la piel de cada sujeto se pueden trascender los factores de riesgo para pensar en términos de interacción y juego social, y descubrir las vulnerabilidades sociales y las lógicas de poder (macro y micro), como también la potencia que subyace en el territorio" (2016, p. 159). El volcadero resulta referencia directa, no solo por parte de distintos profesionales que trabajan en los Centros de Salud de los barrios o en los Centros Comunitarios, sino también por parte de quienes lo circulan y pasan largas jornadas. Los modos de vida sistematizan un conjunto de problemáticas que se concadenan a este habitar el territorio.

Las afecciones respiratorias son identificadas como uno de los principales padecimientos en la niñez, sobre todo en invierno, vinculado a la quema de basura. El humo, como refiere una trabajadora social del Centro de Salud del Barrio Anacleto Medina, produce un olor muy fuerte, generando mucha toxicidad en el ambiente y neblina. Los "gases" y la "pudrición" son materia corriente de quienes asisten a diario. Quienes trabajan en el volcadero también señalan enfermedades y dolencias productos de la exposición e inclemencias del tiempo:

Y... acá creería yo que tanto en el San Martin, como gente que está viviendo en esta mano, en esta parte de la barranca, tiene que sufrir alguna enfermedades de esas porque ya son muchos los años, es décadas bastantes largas que acá fue basural y... por lo tanto, quien diría esto...o sea, en la basura uno contrae cáncer, leucemia, muchas enfermedades también, o sea cuando son crónicos digamos de asma, eh problemas de asma, todas esas cosas (...) [suegra] Ella trabajó en el volcadero y ella venía pero asfixiada por el humo del volcadero. Y un día cayó con neumonía y ahí comenzó con los paf, por el tema 
que le agarró un [¿?], pero todo por el humo del volcadero. iCirujeando! (Poblador Barrio San Martín y trabajador del volcadero)

Estas condiciones afectan a quienes trabajan con la basura y también a quienes viven en las zonas aledañas. La exposición a accidentes es recurrente entre los y las trabajadoras, por la manipulación de la basura y el contacto con elementos cortantes u otro tipo de objetos, como por la proximidad a productos podridos y desechos tóxicos. La entrada y salida de camiones tiene sus implicancias entre quienes esperan la llegada de la mercadería "nueva”, lo cual reconoce dinámicas de poder que asignan "momentos" y tipos de mercaderías. Uno de los acontecimientos que replanteó el propio trabajo fue un accidente de un adolescente de 16 años que se durmió esperando uno de los camiones entrantes al volcadero y lo atropelló, derivando en una fractura de pelvis. Una situación de mucha tristeza, como recupera uno de los trabajadores:

El hijo de una señora que trabaja con nosotros $y$ el chico tiene 16 años y trabaja en el volcadero. Se durmió, trabajando, porque ahí tenés, ellos tienen que cumplir la función de esperar un camión (...) ese chico se durmió para esperar un camión. Por el frío que le dio se tapó con cartones, cuando llegó el camión, no lo sintió. Cuando el camionero hace para atrás para volcarlo, lo pisó. (Poblador Barrio San Martín y trabajador del volcadero)

El suceso del adolescente representa no solo un mero accidente, sino más bien es expresión de un conjunto de problemáticas sociales que él encarna y que pese a que son reconocidas por quienes habitan esos espacios, se esgrimen como inevitables.

La llegada de camiones significa, para muchas personas y familias, la posibilidad de comer ese día. Al basurero municipal, también se suman otros espacios privados donde empresas de supermercados arrojan la mercadería que no sirve para comercializar. La zona, por tanto, es caracterizada por "volcaderos" de distinta procedencia: Entonces la gente que no tiene, que no le alcanza y que no... busca de esa comida. Comida vencida, apretada, rota (Poblador Barrio Balvi).
La necesidad de esperar los camiones, encontrar suministros para alimentarse, la exposición al frío e inclemencias del tiempo nos muestran, de modo sombrío y áspero, que la prevención y la promoción de la salud no son ajenas a las condiciones de vulnerabilidad en la que viven los sujetos: cuando vos estás en crisis y no hay posibilidades de comer bien, y bueno, aparecen las enfermedades, como enfatizaba una de las personas que participa activamente en el Centro Comunitario que linda con el volcadero.

En esta configuración territorial, siguiendo a Marcelo Firpo-Porto (2013), se expresa lo que puede ser referido como territorio urbano de sacrificio. Los barrios periurbanos analizados se configuran como zonas marginalizadas y explotadas de un modelo de desarrollo capitalista que organiza espacialmente en las ciudades diferentes zonas (Bordes; TorresTovar, 2017). El sacrificio evidencia de modo visceral la violencia estructural sobre quienes los habitan. Estas zonas "son expresión de la insustentabilidad de dicho modelo hegemónico de ciudad y desarrollo y se caracterizan por la contaminación industrial del aire, del agua y del suelo, pero también refieren a zonas de sacrificio social donde se niegan los derechos fundamentales y se ejerce violencia para adecuar los espacios a las necesidades de grandes emprendimientos y de los grupos sociales históricamente privilegiados" (Borde; Torres-Tovar, 2017, p. 268).

La vida cotidiana de quienes habitan el territorio los expone a distintas violencias y situaciones que forman parte de circuitos de vulnerabilidad. Los procesos de salud/enfermedad resultan emergente de todo un cúmulo de circunstancias. Las afecciones respiratorias y las alergias no pueden desvincularse de la exposición continua al humo tóxico del volcadero, pero, simultáneamente, son condiciones crónicas vinculadas a los modos de vida y condiciones de vivienda.

En este círculo de vulnerabilidad, la problemática de adicciones y la violencia también son identificadas por los habitantes y trabajadores de la salud. El consumo problemático de sustancias se vincula inexorablemente con circuitos de violencia, de sospecha mutua entre grupos. Cocaína, marihuana, alita de mosca -cocaína cortada con desechos y otros elementos-, drogas sintéticas, constituyen las sustancias más usuales. También 
debemos mencionar el precoz consumo de alcohol en niños/as y jóvenes, como una de las cuestiones más acuciantes.

Las personas que trabajan como profesionales en los Centros de Salud, en los mismos Centros Comunitarios, como los vecinos y habituales trabajadores del volcadero, son conscientes de que las problemáticas de consumo no pueden desvincularse de las condiciones de vulnerabilidad y violencia estructural que persisten. Las políticas o la falta de políticas públicas, la pérdida de empleo, las crisis y la naturalización de un porcentaje cada vez mayor de personas bajo la línea de pobreza resultan un suelo fértil para quienes encuentran en el consumo una "vía de escape", una forma de sortear los flagelos cotidianos y que se "va instalando" con dificultades para revertir.

La violencia también atraviesa los vínculos. A la violencia estructural, también se reconocen la violencia en los vínculos, violencia de género y situaciones de abuso. Las violencias se concadenan y resultan efecto de una multiplicidad de situaciones vividas:

Pero lo que no sale es un montón de cosas que llevan adelante...la violencia, la violencia en el hogar, que a veces el método para calmarlos es cagarlo a palos. Eso no sale. Sale que se droga, pero después hay un montón de otras cosas, todo lo que es el entramado, lo que eso lesiona y fragiliza los vínculos que no sale. Después ¿cómo vos te contenés en un ambiente así? Dónde vos acá, no hay cana que te ayude. Hablaba con una mamá que está desesperada. Le robaron, quiso denunciar y ahora está sufriendo un hostigamiento terrible. De proteger a los hijos. Al hijo de 13 años le gatillaron en la cabeza, amenazas...cosas muy groseras, muy grosas digamos. Mucha vulnerabilidad, de mucho peligro real. (Psicóloga del Centros de Salud del Barrio Anacleto Medina)

Las situaciones de "sospecha", pero también de violencia asociadas al delito -producto a su vez de disputas territoriales, de heridas históricas y controversias familiares-, tienden a profundizarse en la adolescencia, donde también paradójicamente la violencia actúa como modo de contención y generación de vínculos en contextos de fragilidad:
Es una forma de estar la violencia. Una forma de sostenerte. Una forma de dignidad, si se quiere, de poder. Los pibes, los adolescentes se copan mucho con eso. Nadie se identifica con ser un gusano (...) Digamos ser un deshecho. Preferís morir con el fierro en la mano. Esa cosa de la violencia, de sostenerte, como una identidad, si se quiere. No sé... (Psicóloga del Centros de Salud del Barrio Anacleto Medina)

Como reconocen los y las profesionales, el trabajo es también una labor respecto a los prejuicios, el miedo, la impotencia y el dolor, que exige inexorablemente comprender el territorio para elaborar estrategias conjuntas de intervención.

De modo concomitante, la fragilidad en los vínculos, las problemáticas de consumo, los embarazos adolescentes, la crianza de bebés y la necesidad de generar recursos o ingresos para las familias inciden en los aspectos socioeducativos de los niños, niñas, jóvenes y adolescentes: "a la escuela la abandonan temprano”, como refiriera la Trabajadora Social del Centro de Salud. Así, se advierte que las problemáticas socioeducativas se vinculan estrechamente a estos circuitos de vulnerabilidad: Usted pregunta adolescente todo, pero...ese adolescente está juntando basura o haciendo otra cosa (Poblador Barrio Balvi). Y en esta rueda, las experiencias de enfermedad también operan como condicionante de acceso a la educación:

Nuestra compañera acá de la corriente nos comentaba que no podía llevar a los chicos a la escuela porque estaban todos con problemas respiratorios y estaban, no eran uno o dos días, era un mes ponele, con problemas respiratorios, con asma, que no...que estaban...o sea, les costaba respirar y eso le insumía demasiada energía y despúes al otro día estaban tirados, no se podían ni mover, o estaban con un problema infeccioso entonces no se podían, no se podían mover de la casa. Le recomendaba el médico que no vaya a la escuela y bueno... (Integrante del CAAC).

Los territorios o zonas de sacrificio producen cuerpos enfermos, dependientes, frágiles y propensos a generar y vivir situaciones de violencia, que se retroalimentan en un círculo que reproduce 
y naturaliza las inequidades y las vulnerabilidades. Las condiciones estructurales, los contextos de vida marcados por círculos de vulnerabilidad, participan indefectiblemente en los modos de enfermar y morir en los barrios periurbanos estudiados. El territorio conecta con la salud y la vida, en tanto en él se dan los procesos de producción y reproducción social, que son la base de la determinación social. En esta dirección, considerar las contradicciones y alternativas posibles de los grupos y poblaciones constituye un requerimiento básico para comprender los procesos de salud/enfermedad/atención/cuidado desde los saberes y prácticas, desde los modos de vida -como vivencia encarnada anclada a los territorios- de quienes los habitan.

En el caso analizado, no podemos desconocer que el volcadero condensa una serie de problemáticas sociales, como sanitarias. Simultáneamente el volcadero, en un contexto caracterizado por la falta de oportunidades, por situaciones de pobreza e indigencia, resulta una fuente de trabajo fundamental para muchas personas y familias, que se "ocupan" de la basura, que hacen de ella un recurso para vivir o comercializar. Pensar en territorio, implica advertir estas controversias y abordar la promoción de la salud con las contradicciones inherentes, con las posibilidades y alternativas que se presentan para los pobladores de estas zonas de sacrificio, alentando no solo propuestas constructivas, sino también capaces de ser apropiadas y sostenidas por sus habitantes.

\section{Conclusiones}

El territorio como hemos referido resulta más que una localización física-espacial, contenedora de recursos y población. Referimos a situaciones de inequidad y vulnerabilidad, como manifestaciones de la violencia estructural que pueden condensarse bajo la rúbrica de zonas de sacrificio. Las consecuencias y desplazamientos que encarna la existencia de un basural a cielo abierto, las condiciones de trabajo asociadas, pero también de vivienda, medioambientales, como las problemáticas asociadas al consumo de sustancias, a la violencia, a la fragilidad en los vínculos, al acceso a la educación dan cuenta de un complejo entramado.
Atender a la relación territorio y salud, desde la perspectiva de la Medicina Social y Salud Colectiva, promueve considerar estos factores estructurales, sociales, culturales, históricos y políticos para comprender cómo las desigualdades se transforman en inequidades, principios de injusticia que vulneran diversos derechos y que exponen a ciertos colectivos a circunstancias y padecimientos particulares. Así, lejos de pensar la determinación social de la salud desde asociaciones causa-efecto en relación a la salud de la población, el análisis asume la complejidad que encarna el territorio en la producción de subjetividades. Como refiriéramos, desde una perspectiva territorial, no se trata solo de pensar la política sanitaria desde los marcos estructurales, sino también anclada a las propias demandas y necesidades en un proceso de territorialización que involucre los propios modos de vida en sus malestares y problemáticas asociadas.

El volcadero es epicentro de diversos dilemas sociosanitarios, pero también es fuente de recursos para muchas de las personas que "viven" de él, un medio de trabajo y subsistencia -lo cual no excluye reglas, códigos y categorías de trabajadores. Esto exhibe cómo las problemáticas que encarna están lejos de poder ser atendidas desde modelos simplistas, ligadas, por ejemplo, al traslado o eliminación del basural.

Cartografiar los territorios de manera colaborativa constituyó un modo de atender a las complejidades e identificar con los propios habitantes, desde su cotidianeidad, problemáticas sanitarias. Por otro lado, supuso comprender que el territorio encarna relaciones de poder, que se entretejen y atraviesan las prácticas y espacialidades, por ejemplo, en las reglas y códigos de acceso al trabajo en el volcadero o los modos en que los jóvenes, sobre todo, circulan y se ven interpelados por el consumo problemático de sustancias. Como hemos referido, estos aspectos también forman parte de relaciones de poder anudadas a condiciones de vulnerabilidad y violencia estructural, que delinean zonas de sacrificio y procesos de exclusión en las cuales la pérdida de empleo, la pobreza, la falta de políticas públicas, participan en los procesos de determinación social de las desigualdades y, por ende, en los procesos de salud/enfermedad/atención/cuidado. Como bien 
sintetiza Sajama, la gente vive, enferma y muere dependiendo de las condiciones en que trabaja (producción) y vive (reproducción), dinámicas que se dan ligadas al territorio. En esta dirección, la exigibilidad de derechos no puede dirimirse más que colectivamente desde las condiciones posibles, imaginadas, aceptadas, factibles, permitidas, habilitadas, construidas, promovidas para el ejercicio del cuidado, para la producción de la vida y la salud.

\section{Referencias bibliográficas}

ALMEIDA FILHO, N. La ciencia tímida: ensayos sobre deconstrucción de la Epidemiología. Buenos Aires: Lugar Editorial, 2000.

BORDE, E.; TORRES-TOVAR, M. El territorio como categoría fundamental para el campo de la salud pública. Saúde em Debate, Rio de Janeiro, v. 41, n. especial, p. 264-275, 2017.

BREILH, J. Epidemiología Crítica: ciencia emancipadora e interculturalidad. Buenos Aires: Lugar editorial, 2003.

BREILH, J. Informe alternativo sobre la salud en América Latina. Quito: Global HealthWatch, 2010.

BREILH, J. La determinación social de la salud como herramienta de ruptura hacia la nueva salud pública (salud colectiva): epidemiología crítica latinoamericana - raíces, desarrollos recientes y ruptura metodológica. In: SEMINARIO INTERNACIONAL DE LA SALUD PÚBLICA, 8., 2013, Bogotá. Bogotá: Universidad Nacional de Colombia, 2013.

CASALLAS-MURILLO, A. L. La medicina socialsalud colectiva latinoamericanas: una visión integradora frente a la salud publica tradicional. Revista Ciencias de la Salud, Bogotá, v. 15, n. 3 , p. 397-408, 2017. DOI: 10.12804/revistas.urosario. edu.co/revsalud/a.6123

CHIARA, M. (Comp.). Salud, política y territorio en el Gran Buenos Aires. Los Polvorines: Universidad Nacional de General Sarmiento, 2012.

CHIARA M. Territorio, políticas públicas y salud: hacia la construcción de un enfoque multidimensional para la investigación. Revista Gerencia y Políticas de Salud, Bogotá, v. 15, n. 30, p. 10-22, 2016. DOI: 10.11144/ Javeriana.rgyps15-30.tpps

CONICET. Resolución Nro. 2857: lineamientos para el comportamiento ético en las ciencias sociales y humanidades (CSyH). Buenos Aires: CONICET, 2006.

FIRPO-PORTO, M. Injustiça ambiental no campo e nas cidades: do agronegócio químico- dependente às zonas de sacrifício urbanas. In: FIRPO-PORTO, M.; PACHECO, T.; LEROY, J. P. Injustiça ambiental e saúde no Brasil: mapa de conflitos. Rio de Janeiro: Fiocruz, 2013. p. 133-174.

GUBER, R. El salvaje metropolitano: reconstrucción del conocimiento social en el trabajo de campo. Buenos Aires: Paidós, 2005.

HERNÁNDEZ, M. Desigualdad, inequidade injusticia en el debate actual en salud: posiciones e implicaciones. Ciudad de México: Asociación Latinoamericana De Medicina Social, 2008.

INDEC. Provincia de Entre Ríos por departamento. Entre Ríos: Gobierno de Entre Ríos, 2010. Disponível em: https://bit.ly/3se5GHK. Acesso em: 3 out. 2020.

LASSITER, L. E. The Chicago guide to collaborative ethnography. Chicago: Chicago University Press, 2005.

LASSITER, L. E.; GOODALL, H.; CAMPBELL, E.; JOHNSON, M. N. (Ed.). The other side of middletown: exploring Muncie's African American community. Walnut Creek: AltaMira Press, 2004.

LAURELL, A. C. La salud: de derecho social a mercancía. In: LAURELL A. C. (Comp.). Nuevas tendencias y alternativas en el sector salud. Ciudad de México: Universidad Autónoma Metropolitana Unidad Xochimilco, 1994. p. 9-31.

LAWLESS, E. Holy women, wholly women: sharing ministries of wholeness through life stories and reciprocal ethnography. Philadelphia: University of Pennsylvania Press, 1993.

LÓPEZ, S.; MICHELLI, V. (Coord.). Problematizando la salud. Aportes conceptuales y experiencias de trabajo en territorio desde la perspectiva de la medicina social / salud colectiva. La Plata: Universidad Nacional de La Plata, 2016. 
MENÉNDEZ, E. L. Estilos de vida, riesgos y

construcción social. In: MENÉNDEZ, E. L. De sujetos, saberes y estructuras: introducción al enfoque relacional en el estudio de la salud colectiva. Buenos Aires: Lugar Editorial, 2009. p. 37-67.

MOLINA JARAMILLO, A. N. Territorio, lugares y salud: redimensionar lo espacial en salud pública. Cadernos de Saúde Pública, Rio de Janeiro, v. 34, n. 1, p. 1-12, 2018. DOI: 10.1590/o102-311xooo75117
POLO ALMEIDA, P. E. Modos de vida: una categoría esencial en geografía y salud. Buenos Aires: CLACSO, 2016.

RAPPAPORT, J. RODRIGUEZ, M. E. Más allá de la escritura: la epistemología de la etnografía en colaboración. Revista Colombiana de Antropología, Bogotá, v. 43, p. 197-229, 2007.

SPINELLI, H. Volver a pensar en salud: programas y territorios. Salud Colectiva, Lánus, v. 12, n. 2, p. 149-171, 2016. DOI: 10.18294/sc.2016.976

\section{Agradecimientos}

Este artículo fue posible gracias a los diálogos y aportes del Lic. en Enfermería Leandro Alva y de la Lic. en Trabajo Social Teresita Calzia. También es producto de la generosidad de la Dra. Natalia Luxardo y de su trabajo conjunto para la producción de estos resultados. Finalmente, mencionar que el artículo forma parte de una investigación colaborativa mayor sobre desigualdades sociales en salud.

Recibido: 08/10/2020

Re-presentado: 08/10/2020

Aprobado: $21 / 11 / 2020$ 